\title{
AS DOENÇAS RARAS NA ESCOLA UMA INCURSÃO PELA LITERATURA*
}

\author{
Felícia Catana ${ }^{1}$ \\ Célia Ribeiro ${ }^{2 \dagger}$ \\ Anabela Carvalho ${ }^{3 *}$
}

\begin{abstract}
Resumo: Ainda que do ponto de vista da prevalência sejam raras, o impacto das Doenças Raras (DR's) é considerável, quando se verifica que, no seu conjunto, afetam uma percentagem muito significativa da população. Tendo em conta que estas doenças diminuem consideravelmente a qualidade de vida das crianças atingidas, bem como dos seus familiares, os profissionais da educação deverão estar atentos e preparados para lidar com esta realidade.

Relativamente ao impacto da Doença Rara (DR) na vida escolar do aluno, o apoio a prestar beneficiaria com uma visão holística, por parte da equipa educativa, não só das dificuldades e necessidades dos alunos e suas famílias, mas também das suas potencialidades educativas. Estudos recentes preconizam uma intervenção educativa baseada na etiologia, por forma a otimizar o processo de ensino-aprendizagem junto das crianças com DR.
\end{abstract}

Palavras-chave: Doenças Raras, escola, etiologia, intervenção educativa

\footnotetext{
* Este artigo retoma, parcialmente, a revisão da literatura da Dissertação de Mestrado em Ciências da Educação, Especialização em Educação Especial, apresentada por Felícia Catana sob orientação da Professora Doutora Célia Ribeiro e Doutoranda Anabela Carvalho, no Centro Regional das Beiras da Universidade Católica Portuguesa, em 2013.

† Docente de Educação Especial, Mestre em Ciências da Educação, Especialização em Educação Especial, Centro Regional das Beiras, Universidade Católica Portuguesa. E-mail: feliciacatana@sapo.pt

* Professora Auxiliar da Universidade Católica Portuguesa - Viseu. Doutora em Psicologia, área de especialidade em Psicologia Pedagógica. E-mail: cribeiro@crb.ucp.pt

* Psicóloga do Agrupamento de Escolas Viseu Sul, Doutoranda em Psicologia do Desenvolvimento. E-mail: anabela.cruz.carvalho@gmail.com
} 


\title{
RARE DISEASES IN SCHOOL AN INCURSION IN THE LITERATURE
}

\begin{abstract}
Although rare in prevalence, Rare Diseases (RDs) have a considerable impact when we realise that, all together, affect a significant percentage of the population. Considering that these diseases substantially reduce the quality of live of the affected children and their families, education professionals need to be alert and prepared to deal with this reality.

Regarding the impact of the Rare Disease (RD) in the student's academic life, the support provided would benefit from a holistic approach from the education team, concerning not only the students and their families' difficulties and needs, but also their educational potential. Recent studies suggest an educational intervention based on etiology, in order to enhance the teaching-learning process among children with RD.
\end{abstract}

Key words: Rare Diseases, school, etiology, educational intervention

\section{INTRODUÇÃO}

A temática das Doenças Raras (DR's) tem vindo a ser alvo de interesse, naturalmente, por parte de investigadores da área da medicina, mas também de outras áreas, havendo alguns estudos sobre as implicações sociais, económicas, psicológicas e educativas que estas doenças acarretam. Ainda assim, os estudos desenvolvidos nesta área circulam em meios restritos, no plano da investigação científica, fora do alcance das equipas que diretamente intervêm com a criança e a sua família.

Embora cada Doença Rara (DR) se caracterize por um reduzido número de pessoas afetadas, com diferentes especificidades, quando entendidas num vasto conjunto estas patologias representam números alarmantes de pacientes. Estes partilham características e dificuldades semelhantes, representando um elevado nível de sofrimento, tanto para pacientes como para os seus familiares, dadas as graves limitações na participação em atividades do dia a dia, ditadas pela patologia. De facto, apesar de uma DR afetar uma minoria de sujeitos (menos de 1 em cada 2000 nados-vivos), existe uma vasta gama de DR's diferentes (mais de 6000), cada vez mais numerosa, tendo em conta os céleres avanços científicos nesta área.

Estes dados alertaram-nos para os problemas de saúde pública que daqui advêm e, consequentemente, para os problemas sociais, económicos, 
psicológicos, familiares e educativos que estas doenças implicam.

Neste trabalho, revemos os autores que se dedicaram ao estudo das implicações das DR's, sob o ponto de vista educativo. Abordamos, inicialmente, o conceito de DR e percorremos o historial político desta problemática na Europa.

Analisamos, por fim, a parca bibliografia referente às implicações das DR's em contexto escolar. Sendo a DR, na sua génese, uma doença crónica e pervasiva na vida do indivíduo, refletimos acerca de um modelo sistémico sobre o desenvolvimento da criança com doença crónica.

Debruçamo-nos ainda sobre a importância do diagnóstico para a intervenção educativa, com ênfase em estudos recentes que preconizam a intervenção educativa baseada na etiologia, como forma de otimizar o processo de ensino-aprendizagem das crianças com DR.

Pretendemos um melhor entendimento desta problemática em meio escolar, com vista à melhoria das práticas das equipas que intervêm com as crianças e famílias afetadas por DR.

\section{ENQUADRAMENTO TEÓRICO - O QUE SÃO DOENÇAS RARAS?}

De uma forma geral, as fontes consultadas definem DR como aquela que afeta um número limitado de indivíduos, sendo na União Europeia (UE) menos de 1 caso em 2.000 (Dodge et al., 2011; EURORDIS, 2007; Remuzzi \& Garattini, 2008; Schieppati, Henter, Daina, \& Aperia, 2008; Tarniceru, Puiu, Cerbu, \& Serban, 2007; Yaneva-Deliverska, 2011; Zurynski, Frith, Leonard, \& Elliott, 2008). Relativamente ao número destas doenças e dos seus afetados, os autores são unânimes em sublinhar o quão difícil é quantificar, uma vez que a resposta é contextual. Podem variar no tempo e na área geográfica, podendo uma doença ser rara numa região e frequente noutra (Aymé, 2000; Dellve, Samuelsson, Tallborn, Fasth, \& Hallberg, 2006). Para além desta dificuldade, os mesmos autores destacam o facto de todas as semanas serem descobertas cerca de cinco DR's, considerando que não são novas doenças, mas ignoradas até então, por se verificar subdiagnóstico nestas patologias.

De acordo com a Decisão n. ${ }^{\circ}$ 1295/1999/CE do Parlamento Europeu e do Conselho, que adota o primeiro programa de ação comunitária em matéria de DR's, para 1999-2003,

(...) entendem-se como doenças raras, incluindo as de origem genética, as doenças (...) cuja prevalência é tão reduzida que o seu tratamento exige a conjugação de esforços especiais para tentar 
evitar elevadas taxas de morbilidade ou mortalidade perinatal e precoce, bem como uma diminuição considerável da qualidade de vida ou do potencial socioeconómico dos indivíduos. (...) pode considerar-se reduzida uma prevalência (...) inferior a 5 por 10.000 na Comunidade. (Comissão Europeia, 1999, p. 2)

A definição de DR utilizada na UE é diferente noutros países ou nos Estados Unidos da América (EUA), tendo sido estabelecida pelo critério da prevalência. No entanto, a utilização deste critério para definir uma doença como sendo rara levanta vários problemas, pois internacionalmente não há critérios estandardizados, sendo frequente que muitas destas doenças não tenham estudos epidemiológicos (Gaite, Fuentes, Lamuño, \& Álvarez, 2008). Não há sequer uniformidade de critério quanto à taxa de prevalência da mesma doença. Se na UE se aceita a prevalência de 5 para 10.000, já na Austrália é 1/10.000, no Japão 4/10.000 e nos EUA 7,5/10.000. Gaite e colaboradores (2008) acrescentam que os próprios critérios da Classificação Internacional de Doenças (CID) poderão não se adequar a DR's específicas, sendo usual classificá-las de "outras". Também Schieppati e colaboradores (2008) admitem que a utilização da CID na maioria dos países não é conveniente para as DR's, sendo a falta de um sistema de codificação, universalmente reconhecido, um obstáculo para o registo de doentes em bases de dados nacionais e internacionais que permitiriam avaliar os efeitos económicos e sociais das DR's.

Estas dificuldades de classificação e registo em bases de dados foram, aliás, focadas no Background Paper on Orphan Diseases for the "WHO Report on Priority Medicines for Europe and the World", documento que justifica a problemática das DR's como uma das prioridades, a nível da medicina, na Europa e no Mundo, segundo a Organização Mundial de Saúde (Weely \& Leufkens, 2004).

Um estudo divulgado pela Comissão Europeia, em 2011, revela que os portugueses apresentam uma fraca consciencialização sobre DR's e poucos conhecimentos sobre doenças raras específicas, relativamente à média comunitária (European Comission, 2011). Este Eurobarómetro indica que os europeus têm, na generalidade, um conhecimento razoável sobre estas doenças (Yaneva-Deliverska, 2011). No entanto, e apenas em relação à população portuguesa inquirida, apenas $50 \%$ conhece a definição correta para este tipo de patologias e, sobre DR's concretas, os portugueses revelam um desconhecimento bem superior comparativamente à média europeia. Não é de estranhar que, nesta linha, 51\% dos inquiridos concorde que Portugal tem muitos outros problemas de saúde para fazer das DR's uma prioridade, tendo a média europeia atingido apenas os $39 \%$. 
Yaneva-Deliverska (2011) particulariza a exceção de Portugal, quando a maioria dos inquiridos, em cada Estado-Membro, julga ser totalmente justificado que se reúnam esforços para apoiar as famílias afetadas por uma DR. Esta autora considera que há uma disposição generalizada entre os europeus para apoiar questões relacionadas com esta temática, ao contrário dos portugueses, mas adianta que se trata mais de instinto e empatia, do que de um facto baseado no conhecimento. Daí julgar ser necessária uma política futura acompanhada de educação e sensibilização para o reconhecimento e visibilidade das DR's, de forma a divulgar a sua existência e implicações médicas, sociais, educativas e psicológicas entre a população em geral.

A Recomendação do Conselho da UE alerta para números muito significativos de pacientes afetados por DR's:

Estima-se que existam hoje entre 5.000 e 8.000 doenças raras diferentes, que afectam entre $6 \%$ e $8 \%$ das pessoas no decurso das suas vidas. Por outras palavras, embora as doenças raras sejam caracterizadas por uma baixa prevalência para cada uma delas, o número total de pessoas por elas afectadas na UE situa-se entre 27 a 36 milhões. A maioria dessas pessoas sofre de doenças menos frequentes que afectam uma em cada 100000 pessoas ou menos. Esses pacientes encontram-se particularmente isolados e vulneráveis. (Conselho da União Europeia, 2009, p. C 151/7)

O desconhecimento sobre as DR's faz-nos pensar que "só acontece aos outros", mas em matéria de DR's a única certeza que temos é que atingem qualquer pessoa, mesmo que não se manifestem logo no nascimento. A este propósito, Tavares (1996) sublinha que:

(...) ninguém está isento de alterações e, numa extensa variedade de genótipos cada um de nós transporta um certo número de deficiência e imperfeições genéticas. Algumas não têm expressão significativa e são imperceptíveis, outras podem condicionar alterações graves num vasto leque de fenótipos variantes intermédios. (p. 261)

Para termos uma ideia do número das doenças genéticas com implicações no desenvolvimento cognitivo, há autores que consideram uma causa genética para cerca de $1 / 3$ dos casos com dificuldade intelectual (Heikura et al., 2005, citado por Wulffaert, 2010), tendo sido identificadas por volta de 1500 síndromes com associação a este défice (Oliver \& Hagerman, 2007, citado por Wulffaert, 2010).

Por seu turno, as estimativas apontam para $1 \%$ de crianças em idade 
escolar que são afetadas por deficiências, tendo metade destes casos uma causa pré-natal (Guillem, Cans, Robert-Gnansia, Aymé, \& Jouk, 2008). O estudo epidemiológico de Guillem e seus colaboradores (2008), sobre a contribuição das DR's nas deficiências graves de crianças com 7 anos de idade, realçou que pelo menos $1 / 4$ das deficiências graves mentais, sensoriais e/ou neuromusculares, classificadas através da Classificação Internacional de Funcionalidade (CIF), se deve a DR's.

O gráfico I ajuda-nos a visualizar os resultados deste estudo, em que destacamos a cor mais clara (deficiências com etiologia devida a DR's) e a mais escura (de origem desconhecida).

\section{Gráfico I}

Proporção de crianças com Doença Rara de acordo com o tipo de deficiência mental, sensorial e/ou neuromuscular

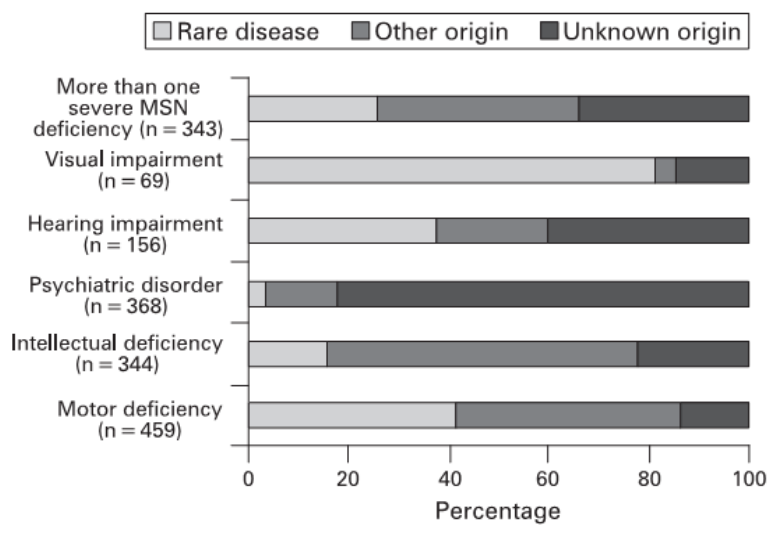

(Guillem et al., 2008, p. 117)

É importante salientar que a taxa de DR's pode estar subestimada, uma vez que, em quase $40 \%$ dos casos estudados a etiologia é desconhecida, o que significa que possa haver outras DR's não diagnosticadas.

\subsection{As mesmas dificuldades em especificidades diferentes}

Vários autores referem que nas especificidades diferentes, as DR's partilham as mesmas dificuldades, o que nos ajuda a compreendê-las (Aymé, 2000; Dellve et al., 2006; Dodge et al., 2011; EURORDIS, 2007; Tarniceru et al., 2007; Taruscio \& Cerbo, 1999; Yaneva-Deliverska, 2011; Zurynski et al., 2008). Dessas características transversais à 
problemática em causa, destacamos:

- as DR's são crónicas, progressivas, degenerativas e muitas vezes implicam risco de vida. Apesar de diferirem na severidade, a esperança média de vida é, na generalidade, significativamente reduzida;

- algumas DR's são compatíveis com uma vida normal, se forem diagnosticadas, geridas e tratadas atempada e adequadamente, o que nem sempre ocorre;

- são de difícil diagnóstico, verificando-se atraso ou falta de acesso a um correto diagnóstico, com todas as consequências nefastas que daqui advêm;

- devido à falta de conhecimento científico generalizado entre os especialistas, nem sempre a informação de qualidade é assegurada, principalmente na própria língua dos afetados;

— são incapacitantes, pois há comprometimento da qualidade de vida dos pacientes, pela falta ou perda de autonomia;

- o acesso a cuidados de saúde de qualidade e a recursos terapêuticos inovadores é difícil, labiríntico e desigual.

- não têm cura efetiva, sendo o tratamento geralmente impotente;

- 75\% das DR's afetam crianças, sendo que em $30 \%$ dos pacientes a morte ocorre antes dos 5 anos de idade;

- $80 \%$ têm origem genética, envolvendo um ou mais genes ou aberrações cromossomáticas, decorrentes de fatores hereditários ou de situações de novo, sendo as restantes o resultado de infeções, alergias ou causadas pelo ambiente.

Depreendemos, pelo exposto, que os pacientes se confrontam com inúmeras dificuldades que advêm diretamente da raridade destas patologias (Yaneva-Deliverska, 2011).

Por este motivo, as DR's representam um problema de saúde pública significativo, com aspetos peculiares que deverão ser reconhecidos no sentido de se organizar uma estratégia de intervenção adequada (Taruscio \& Cerbo, 1999).

O peso deste problema de saúde pública foi avaliado num questionário dirigido a 2500 doentes com doença crónica, em que cerca de $8 \%$ eram raras (Weely \& Leufkens, 2004). Os resultados indicam que os pacientes com DR's apresentam as piores experiências em termos de perda de oportunidades sociais, económicas e de cuidados médicos, comparativamente a doenças crónicas mais comuns. Daí que a uma baixa prevalência não corresponda, de todo, um baixo impacto (Zurynski et al., 2008). Zagalo-Cardoso (2001) realça o facto de que, apesar de cada 
doença genética ser "relativamente rara, a sua importância é muito superior ao que seria de supor" (p. 211).

\subsection{Historial político das DR's na Europa - do raro a uma abordagem global}

Ao contrário dos EUA, onde o suporte legislativo para medicamentos órfãos ocorreu em 1983 (denominado Orphan Drug Act, sob os auspícios da National Organization for Rare Disorders - NORD), o foco nas DR's a nível europeu é um fenómeno relativamente recente. Apesar de alguns países nórdicos (como a Suécia, Dinamarca e Noruega) terem iniciado por livre iniciativa o apoio às necessidades dos pacientes, quer a nível de diagnóstico, quer de cuidados, as autoridades de saúde e os políticos europeus ignoraram as questões relacionadas com as DR's até recentemente. Só na viragem do milénio, foram regulamentadas na UE leis similares ao Orphan Drug Act, nomeadamente o Regulamento (EC) 141/2000 do Parlamento Europeu e do Conselho sobre medicamentos órfãos, em 16 de dezembro de 1999, permitindo que as DR's passassem a ser consideradas uma das áreas prioritárias, a nível da investigação e dos programas de saúde pública, nos vários países europeus (Guillem et al., 2008).

Aymé e Schmidtke (2007) descrevem algumas das principais iniciativas em termos de políticas de saúde para DR's na UE, fazendo alusão ao primeiro Programa de Ação Comunitária para DR's. Trata-se da Decisão n. ${ }^{\circ}$ 1295/1999/CE do Parlamento Europeu e do Conselho, adotado para 1999-2003, com vista à divulgação do conhecimento e à facilitação do acesso à informação sobre estas doenças (Comissão Europeia, 1999). No programa seguinte de saúde pública da UE (Decisão n. ${ }^{\circ}$ 1786/2002/CE), delineado para 2003-2008, as DR's continuaram a ser uma prioridade, bem como no contexto do programa comunitário previsto para 2008-2013 (Decisão n. ${ }^{\circ}$ 1350/2007/CE).

Este esforço conjunto permitiu, nos últimos anos, o crescente interesse científico e institucional por estas questões, com a adoção de medidas políticas para apoiar e responder às necessidades dos pacientes (Gaite et al., 2008). Vários países europeus, entre os quais Portugal, elaboraram programas políticos específicos, movidos por associações de pacientes e seus familiares (no caso português, por exemplo, a Associação Raríssimas) que paulatinamente foram pressionando as autoridades políticas para a assunção de compromissos legais. O Programa Nacional de Doenças Raras, datado de 2008, surge no contexto do Plano Nacional de Saúde 
2004-2010 que relativamente às DR's refere: “As doenças genéticas raras, causadas por alterações existentes no genoma, são situações crónicas, habitualmente congénitas, que se podem repetir nas famílias e, embora tenham reduzida prevalência no período pós-natal, demonstradamente contribuem para uma importante fatia da morbimortalidade durante os primeiros 18 anos de vida" (Ministério da Saúde, 2004, p.73).

A generalidade dos autores consultados admite a dificuldade em desenvolver uma política de saúde pública destinada a cada DR, postulando antes a necessidade de uma abordagem global, em vez de fragmentada (Aymé \& Schmidtke, 2007; Barrio \& Castro, 2008). De facto, reconhecer as DR's como tema de política pública é duplamente justificado, dada a gravidade da situação individual dos doentes e a urgência coletiva que estas doenças representam, quando encaradas no seu todo (Aymé, 2000). Graças aos programas de índole política, tem sido possível vários projetos desenvolverem as suas atividades a nível da UE, como a Orphanet, EURORDIS e vários centros de referência, entretanto criados na Europa.

\section{AS DOENÇAS RARAS EM CONTEXTO ESCOLAR}

Devido aos avanços médicos e tecnológicos, o número de alunos com doença crónica tem vindo a aumentar nas escolas, sendo agora crónico o que era considerado terminal (Shaw, Glaser, Stern, Sferdenschi, \& McCabe, 2010).

De facto, doenças que há duas décadas implicavam uma permanência e tratamento apenas em local hospitalar são agora monitorizadas pela família e pela escola. Por este motivo, McCabe e Shaw (2010) consideram que o papel das escolas se modificou, tornando-se mais flexíveis no desenvolvimento e implementação de programas de apoio aos alunos com doença e com deficiência.

No desenvolvimento da vida de uma criança, e por interferir em todas as atividades quotidianas, a doença altera e afeta o seu processo de socialização e de formação, condicionando, em muitos casos, a continuidade escolar e as aprendizagens.

A orientação escolar de crianças com DR não difere, em princípio, daquela das outras crianças com problemáticas mais comuns. No entanto, tal como referem Barco, Domínguez, Solana, Vásquez e Díaz (2008), por haver uma enorme diversidade de DR's com diferentes tipologias, não se podem estabelecer linhas educativas comuns, sendo necessária a diferenciação no processo de avaliação e das aprendizagens. 
No entanto, a raridade e o seu lado atípico complicarão a tarefa do professor/educador, uma vez que será difícil delimitar as necessidades da criança e definir a intervenção educativa apropriada, à luz da atual legislação que obriga à classificação da funcionalidade dos alunos. Referimo-nos ao Decreto-Lei n. ${ }^{\circ} 3 / 2008$, de 7 de janeiro, que assegura legalmente o direito de todas as crianças serem acolhidas pela escola, independentemente das suas condições físicas, intelectuais, sociais e emocionais, postulando uma resposta educativa adequada à especificidade de cada caso.

Quando um aluno com DR ingressa na escola, é imprescindível que o professor esteja a par das suas características, mas também das suas necessidades (Barragán, 2010). No entanto, e dado o problema da falta de informação comum nestas patologias, Gaite e colaboradores (2008) sublinham que há motivo de preocupação pela inexistência de pessoal adequadamente preparado para atender as crianças com DR.

De facto, a informação de que os professores dispõem não facilita a implementação de pedagogias e respostas diferenciadas, já que aquela não se encontra em formato acessível e a linguagem técnica com que é apresentada tem pouca relevância em termos educativos (McCabe \& Shaw, 2010).

Tendo em conta a pouca difusão de estudos sobre a vida escolar de crianças com DR, julgamos que o aproveitamento de investigações na área das doenças crónicas será importante para compreender esta realidade, até porque as DR's também se caracterizam pelo carácter crónico nas suas manifestações. Os estudos efetuados na área da doença crónica sublinham que o impacto da doença coexiste com repercussões no âmbito educativo e social, sendo frequentes problemas de comportamento, emocionais e fracasso escolar nas crianças afetadas (Thies, 1999).

Shaw e colaboradores (2010) descrevem as causas do insucesso escolar a que estes alunos estão votados, devido ao absentismo, stress e ansiedade, bem como à medicação que, muitas vezes, provoca efeitos secundários que afetam a aprendizagem (letargia, agitação, fadiga, dificuldade de concentração, náusea, labilidade emocional, tremores, entre outros).

$\mathrm{Na}$ verdade, para além das dificuldades académicas, os alunos com doença crónica enfrentam problemas psicológicos, sociais e emocionais, tornando difícil a integração social, principalmente com os pares (Shaw et al., 2010). Estudos de Mukherjee, Ligthfoot e Sloper (2000) demonstram que os alunos com doença crónica apresentam maior risco que os seus pares de desenvolver problemas psicossociais e académicos. Para Shiu 
(2001), será então importante compreender as necessidades de alunos com doença crónica, de forma a proporcionar equidade de oportunidades educativas. Esta autora preconiza que os programas educativos desenvolvidos para estes alunos deverão prever não só as necessidades académicas, mas também as sociais e emocionais.

Shiu (2005) estudou as perceções de 121 pais sobre questões relacionadas com a vida escolar dos filhos com doença crónica, incluindo várias DR's. Os alunos que apresentam um nível elevado de absentismo, devido à sua doença, são também aqueles que a escola menos apoia e com os quais perde o contacto. $\mathrm{Na}$ mesma linha, os alunos com um suporte social menos favorecido, em termos de amizades com os pares, são também aqueles que os pais consideram infelizes na escola.

No estudo levado a cabo por Oeseburg, Jansen, Reijneveld, Dijkstra e Groothoff (2010), foram analisadas as doenças crónicas em adolescentes com dificuldade intelectual, considerando que aquelas aumentam largamente a probabilidade de problemas emocionais e comportamentais. Para estes autores, os professores tendem a atribuir problemas emocionais e comportamentais à dificuldade intelectual dos alunos e não a consequências da doença crónica, podendo conduzir à manutenção e agravamento dessas manifestações, já que não são utilizadas intervenções efetivas para prevenir os sintomas da doença. Para Oeseburg e colaboradores (2010), os professores terão aqui um papel fundamental, no conhecimento das implicações da doença crónica em alunos com dificuldade intelectual.

Um dos poucos estudos que aborda, para além de outras questões, a vertente escolar nas crianças com doença genética, foi levado a cabo por Gallo, Hadley, Angst, Knafl e Smith (2008). Estes autores constataram que cerca de metade dos pais inquiridos exprimiram preocupação com o desempenho escolar da criança, devido ao absentismo e problemas na aprendizagem relacionados com a sua doença genética. O bem-estar físico e psicológico foi também apontado pelos pais como motivo de preocupação, já que os filhos se sentem diferentes dos outros nas restrições alimentares e nas atividades físicas, sendo mesmo humilhados pelos pares, dada a visibilidade da doença.

Com base nestas preocupações, é determinante o aumento de comunicação entre pais, escola e profissionais de saúde, possibilitando uma melhor compreensão em relação às doenças das crianças (Gallo et al., 2008). Para Thies (1999), a dificuldade em gerir as necessidades das crianças com doença crónica prende-se com o facto de estas se encontrarem na interseção entre os sistemas de saúde e da educação, 
habituados a trabalhar em separado.

Neste sentido, as práticas colaborativas entre os profissionais e a família são importantes na intervenção com crianças com doença crónica (Power, DuPaul, Shapiro, \& Kazak, 2003). Estes autores propõem o modelo apresentado na figura I que inclui as conexões entre os sistemas com variáveis que afetam o desenvolvimento da criança com doença crónica.

\section{Figura I}

Sistemas e interações intersistémicas para a promoção da saúde das crianças com doença crónica

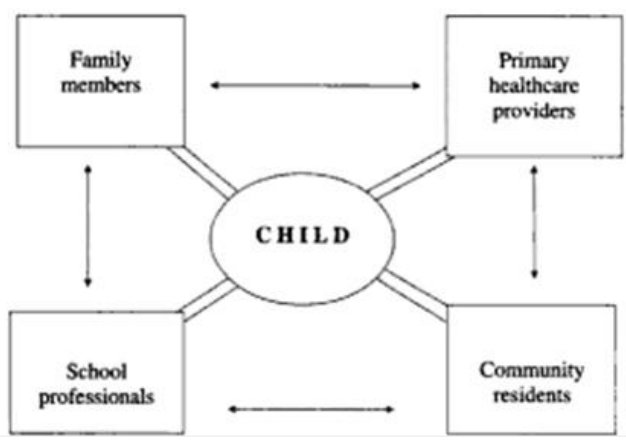

(Power, DuPaul, Shapiro, \& Kazak, 2003, p. 154)

No centro deste modelo, encontra-se a criança ligada pelas linhas paralelas aos principais sistemas que afetam o seu desenvolvimento: a família, a escola, os profissionais de saúde e a comunidade. As setas bidirecionais descrevem as relações de parceria entre aqueles sistemas.

Power (2006) reflete sobre as interações sistémicas, nomeadamente entre a família e os profissionais de saúde, referindo que os cuidados centrados na família são essenciais na intervenção com a criança. Por outro lado, a interação família-escola, apoiada numa relação colaborativa, é importante para promover o sucesso académico e social da criança. Para este autor, nos casos de doença complexa, o conflito entre pais e professores pode eclodir, sobretudo se estes últimos não compreenderem as necessidades especiais da criança.

Já a relação entre o sistema escolar e de saúde nem sempre é a mais coordenada, sendo uma barreira significativa nos casos de limitações devido a doença crónica (Power, 2006). 
A compreensão destas relações sistémicas afigura-se-nos importante para determinar quais os fatores que estão a comprometer o bem-estar e o desenvolvimento da criança. Para este autor, as práticas colaborativas entre os sistemas constituem, então, um desafio para promover a saúde, resolver conflitos e reduzir riscos.

\section{INTERVENÇÃO EDUCATIVA BASEADA NA ETIOLOGIA}

A importância do diagnóstico na área das DR's, como potenciador da intervenção educativa, tem sido alvo de interesse crescente nos últimos anos. Barco e colaboradores (2008) referem que um diagnóstico precoce da DR da criança permite uma intervenção educativa mais adequada e um melhor desenvolvimento posterior.

Para Gilmore e Campbell (2006), conhecer a etiologia das dificuldades da criança possibilita um melhor conhecimento sobre a mesma e fornece a base para uma efetiva intervenção. A partilha de conhecimento sobre DR's fora da literatura médica é, segundo as autoras, uma forma de melhor divulgar e compreender estas patologias e, consequentemente, permitir uma resposta mais apropriada e sensível na educação, na prestação de serviços e no apoio à família. Gilmore (2009) alerta os profissionais para o facto de, muitas vezes, os casos descritos sobre cromossomopatias raras evidenciarem apenas as consequências negativas no desenvolvimento, não sendo relatados os casos positivos ou os pontos fortes, pelo que os profissionais deverão ter em conta as limitações deste tipo de fontes.

As autoras contrapõem também as implicações educativas no desenvolvimento das perturbações raras às mais comuns, como a Trissomia 21 ou do Espetro do Autismo, que têm já uma base de literatura considerável para orientar as famílias, professores e outros profissionais que trabalham com crianças com este tipo de problemas. No caso de doenças sem diagnóstico ou de diagnóstico raro, consideram que se torna mais difícil determinar as respostas interventivas mais apropriadas.

De facto, enquanto para algumas síndromes relativamente comuns, os professores veem a sua intervenção facilitada, Rezazadeh e Shaw (2010) consideram que para a maior parte das doenças raras a categorização em "outros problemas de saúde" conduz os profissionais da educação a intervirem baseando-se no comportamento e adaptando programas educativos dirigidos a crianças com défices cognitivos.

Atualmente, as perturbações são encaradas tendo em conta os seus défices funcionais, ou seja, as consequências na vida do indivíduo (Rezazadeh \& Shaw, 2010). Em contrapartida, os avanços na área da 
genética têm possibilitado predizer os comportamentos e a capacidade cognitiva de algumas síndromes, podendo estas informações guiar educadores e professores para distinguirem essas perturbações e designarem intervenções educativas apropriadas, com um determinado alvo, isto é, focadas em estratégias bem delineadas para colmatar um problema específico (Rezazadeh \& Shaw, 2010).

Uma intervenção precoce, baseada no conhecimento dos fenótipos comportamentais de síndromes genéticas específicas, reduziria, mais tarde, o impacto negativo no desenvolvimento. No entanto, para os autores citados, esta abordagem ainda não foi transferida do campo da investigação genética para a educação, não sendo possível uma intervenção preventiva. Esta nova perspetiva abriria o caminho para a Educação Especial do século XXI.

Autores como Hodapp, DesJardin e Ricci (2003) defendem a ideia que "etiology does matter" (p. 152). Estes investigadores alertam que, em muitas síndromes genéticas, o tipo de doença que afeta a criança é determinante para o comportamento que esta manifesta ou virá a manifestar e, consequentemente, para tomadas de decisão educativas apropriadas. No mesmo sentido, Hodapp e Ricci (2002) preconizam intervenções educativas baseadas na etiologia, usando os fenótipos comportamentais, nos seus pontos fracos e fortes, que deverão ser tidos em conta no âmbito da Educação Especial.

Neste contexto, em doenças genéticas específicas, a intervenção baseada na etiologia permite antecipar e responder melhor aos problemas que estas situações complexas vão desvendando (Hodapp \& Fidler, 1999). Embora se reconheça variabilidade dentro de uma mesma perturbação, considera-se também que existe uma maior probabilidade de um fenótipo comportamental particular se manifestar.

Esta ideia recente é também veiculada pela American Association on Intellectual and Developmental Disabilities (AAIDD, 2010), que realça o papel da etiologia em várias doenças genéticas, como por exemplo, nas síndromes Williams, Prader-Willi, Velocardiofacial, Rubinstein-Taybi, Smith-Magenis ou Angelman, tendo em conta os fenótipos comportamentais que estão frequentemente presentes e associados a estas DR's. Esta entidade considera que "If a behavioral phenotype is known, this information may be useful for all of the team members, including teachers, therapists, counselors, and family members" (AAIDD, 2010, p. 72).

Os estudos de Lozano, Ferreras, Gomariz e Bogdanovitch (2010) conduzem-nos à análise cognitiva e comportamental de quatro DR's - 
síndromes de Rett, Cri-du-Chat, X-Frágil e de Williams - e a sua repercussão no contexto escolar e familiar.

Especificando abordagens para cada síndrome, estes autores sublinham a importância do diagnóstico clínico da síndrome X-Frágil, confirmado molecularmente, para planear estratégias educativas eficazes. Nesta síndrome, se o ambiente educativo não for controlado na idade pré-escolar, tanto em casa como na escola, os problemas de comportamento cristalizarse-ão no desenvolvimento da criança, sendo depois difícil a sua modificação.

A necessidade de um diagnóstico e intervenção precoce é igualmente referida pelos autores a propósito da síndrome de Williams, uma vez que as habilidades motoras e visuoespaciais se deterioram de forma severa com o passar do tempo, devendo ser aproveitados os pontos fortes que caracterizam estas crianças na componente social e nas habilidades verbais expressivas. Pelo contrário, na síndrome de Rett já não há benefício com a aplicação das mesmas estratégias educativas, dados os diferentes padrões cognitivos e comportamentais.

A propósito da síndrome Cri-du-Chat, estes investigadores recomendam que desde cedo se utilizem técnicas de estimulação cognitiva e se estabeleça um canal de comunicação eficaz, pois a maior parte destas crianças não chega a falar. O objetivo será prevenir comportamentos disruptivos, tão típicos nesta patologia, como as agressões e as autolesões, por incapacidade de comunicarem os seus desejos.

De forma a obter formação sobre as características das crianças com doenças genéticas, bem como os seus pontos fortes para deles fazer o melhor uso e adaptar o currículo a lecionar, Rezazadeh e Shaw (2010) sugerem que os professores frequentem conferências e workshops. Recomendam ainda que deverão manter contactos próximos com os pais, de forma a assegurar as necessidades médicas da criança e sua monitorização na escola.

No caso das DR's, e ao contrário da tendência generalizada, são vários os autores que se referem aos pais como experts na doença dos seus filhos. Para Hodapp e colaboradores (2003), os pais representam, muitas vezes, o único elemento entre a equipa educativa que detém experiência e conhecimento sobre uma DR específica. Estes autores recomendam que os profissionais que intervêm na educação devem valorizar a perícia adquirida pelas famílias, já que o conhecimento que têm sobre a criança e a sua síndrome rara constitui uma mais-valia, otimizando toda a intervenção em curso. Solovitch (2001, citado por Skinner \& Schaffer, 2006, p. 23), refere-se aos pais como citizen scientists, cujo conhecimento 
não deve ser descurado pelas equipas que intervêm com a criança.

\section{CONCLUSÃO}

A revisão da literatura permitiu enquadrar teoricamente o tema em estudo, conduzindo-nos a uma visão abrangente das implicações da DR na vida escolar da criança.

Conhecemos a definição e principais características das DR's, bem como as dificuldades partilhadas por pacientes e seus familiares que levaram o poder político europeu, à imagem dos EUA, a intervir nesta questão. Percebemos a razão pela qual estas patologias são consideradas um problema de saúde pública, exigindo esforços e respostas políticosociais concertadas, no apoio às necessidades das pessoas afetadas.

Interessámo-nos particularmente pelas implicações que as DR's têm em contexto escolar. $\mathrm{Na}$ realidade, a escola representa um fator muito importante para o desenvolvimento integral de Todos, e da criança com DR em particular, assumindo-se como um espaço educativo de interação social de eleição, onde aquela passa grande parte do seu tempo.

O desenvolvimento harmonioso da criança com doença dependerá da interação de todos os sistemas que com ela lidam, sendo determinante a colaboração entre a família-escola-hospital-comunidade. Partilhar a educação de uma criança com DR implica uma efetiva comunicação entre estes sistemas, em que todos os elementos da equipa interdisciplinar são reconhecidos como importantes para o seu bem-estar. Esta colaboração e partilha de informação não se deverá centrar apenas nas dificuldades inerentes à DR, mas também nos aspetos positivos que se destacam no desenvolvimento da criança.

Os estudos existentes são escassos e pouco difundidos, mas conduziram-nos a uma nova perspetiva de intervenção educativa baseada na etiologia. Muitos dos comportamentos verificados nas crianças com síndromes raras deve-se à etiologia da doença e não a meras questões temperamentais. Esta perspetiva, que deveria ser explicada pela equipa clínica, poderá ajudar os pais e os professores a compreenderem melhor a DR da criança e a reconhecerem as suas potencialidades educativas.

Consideramos que estamos nos primórdios da Educação Especial do século XXI. A transposição e a difusão dos conhecimentos da área da genética para a área da educação constituem os grandes desafios no apoio educativo a crianças com DR.

Entendemos também que a condição singular da DR de um aluno deveria desencadear no professor um especial cuidado e atenção, incutindo 
no grupo turma e na escola os valores da proteção, da entreajuda e da importância da diversidade para o crescimento harmonioso de cada um.

A mudança de atitudes a nível pessoal, a visão integradora da problemática das DR's, bem como a colaboração e comunicação entre os sistemas que envolvem a criança, são entendidas, então, como uma base fundamental para um atendimento educativo de qualidade às crianças com DR.

\section{FONTES E BIBLIOGRAFIA}

AAIDD. (2010). Intellectual Disability: Definition, Classification and Systems of Supports. (11th ed.) Washington DC : AAIDD.

Aymé, S. (2000). Les injustices de la naissance. Paris: Hachette Littératures.

Aymé, S., \& Schmidtke, J. (2007). Networking for rare diseases: a necessity for Europe. Bundesgesundheitsbl - Gesundheitsforsch Gesundheitsschutz, 50 (12), 1477-1483.

Barco, P. A., Domínguez, M. C., Solana, E. G., Vásquez, I. M., \& Díaz, I. P. (2008). Manual de atención al alumnado com necesidades específicas de apoyo educativo por padecer enfermedades raras y crónicas, 9. Andalucia: Consejería de Educación de Junta de Andalucia.

Barragán, G. M. (2010). Enfermedades raras. Paiderex Revista Extremeña de Formación y Educación, 1 (3), 49-53

Barrio, J. A., \& Castro, A. (2008). Infraestructura y recursos de apoyo social, educativo y sanitario en las enfermedades raras. An. Sist. Sanit. Navar., 31 (2), 153-163.

Comissão Europeia. (1999). Decisão n. ${ }^{o}$ 1295/1999/CE do Parlamento Europeu e do Conselho. Acedido em 1 de dezembro de 2011, em: http://ec.europa.eu/health/archive/ph_overview/previous_programme/ rare_diseases/raredis_wpgm99_pt.pdf

Conselho da União Europeia. (2009). Recomendação do Conselho relativa a uma acção europeia em matéria de doenças raras, pp. 151/7-151/10. Acedido em 1 de dezembro de 2011, em: http://eurlex.europa.eu/LexUriServ/LexUriServ.do?uri=OJ:C:2009:1 51:0007:0010:PT:PDF

Dellve, L., Samuelsson, L., Tallborn, A., Fasth, A., \& Hallberg, L. (2006). Stress and well-being among parents of children of rare diseases: a 
prospective intervention study. Journal of advanced nursing, 53 (4), 392-402.

Dodge, J. A., Chigladze, T., Donadieu, J., Grossman, Z., Ramos, F., Serlicorni, A., Siderius L., Stefadinis CJ, Tasic V., Valiulis A., \& Wierzba J. (2011). The importance of rare diseases: from the gene to society. Arch Dis Child, 96 (9), 791-792.

European Comission. (2011). Special Eurobarometer 361 - European Awareness of Rare Diseases. Acedido em 1 de dezembro de 2011, em: http://ec.europa.eu/health/rare_diseases/docs/ebs_361_en.pdf

EURORDIS. (2007). What is a Rare Disease?Acedido em 1 de dezembro de 2011, em: http://www.eurordis.org/sites/default/files/publications/Fact_Sheet_R D.pdf

Gaite, L., Fuentes, M. G., Lamuño, D. G., \& Álvarez, J. L. (2008). Necesidades en las enfermedades raras durante la edad pediátrica. An. Sist. Sanit. Navar, Supl. 2, 165-175.

Gallo, A. M., Hadley, E. K., Angst, D. B., Knafl, K. A., \& Smith, C. A. (2008). Parent's Concerns About Issues Related to Their Children's Genetic Conditions. JSPN, 13 (1), 4-14.

Gilmore, L. (2009). Rare chromosome disorders and their developmental consequences. Intellectual Disability Australasia, 30 (4), 3-7.

Gilmore, L., \& Campbell, M. (2006). "Give me a name for what is wrong with him": A case study of rare chromosome disorder. Australian Journal of Guidance and Counselling, 16 (2), 225-232.

Guillem, P., Cans, C., Robert-Gnansia, E., Aymé, S., \& Jouk, P. S. (2008). Rare diseases in disabled children: an epidemiological survey. Arch Dis Child, 93, 115-118.

Hodapp, R. M., \& Fidler, D. J. (1999). Special Education and Genetics: Connections for the 21st century. Journal Special Education, 33 (3), 130-137.

Hodapp, R. M., \& Ricci, L. A. (2002). Behavioural phenotypes and educational practice: The unrealized connection. In G. O'Brien (Ed.), Behavioural phenotypes in clinical practice (pp. 137-151). London: MacKeith Press.

Hodapp, R. M., DesJardin, J. L., \& Ricci, L. A. (2003). Genetic Syndromes of Mental Retardation: Should They Matter for the Early Interventionist? Infants $\varepsilon$ Young Children, 16 (2), 152-160.

Lozano, M. P., Ferreras, A. P., Gomariz, M. J., \& Bogdanovitch, A. P. (2010). Rasgos conductales y cognitivos de los síndromes Rett, Cridu-Chat, X-Frágil y Williams. Liberabit, 16, 39-50. 
McCabe, P. C., \& Shaw, S. R. (2010). Genetic and Acquired Disorders: Current Topics and Interventions for Educators. Thousand Oaks, CA: Corwin.

Ministério da Saúde. (2004). Plano Nacional de Saúde 2004-2010. Acedido em 12 de dezembro de 2011, em: http://www.dgsaude.minsaude.pt/pns/media/pns_vol2.pdf

Mukherjee, S., Ligthfoot, J., \& Sloper, P. (2000). The inclusion of pupils with a chronic health condition in mainstream school: what does it mean for teachers? Educational Research, 42 (1), 59-72.

Oeseburg, B., Jansen, D. E., Reijneveld, A., Dijkstra, G., \& Groothoff, W. (2010). Limited concordance between teachers, parents and healthcare professionals on the presence of chronic diseases in IDadolescents. Research in Developmental Disabilities, 31, 16451651.

Power, T. J. (2006). Collaborative practices for managing children's chronic health needs. In L. Phelps (ed.), Chronic health related disorders in children: collaborative medical and psychoeducational interventions (pp. 7-23). Washington DC: American Psychological Association.

Power, T. J., DuPaul, G. J., Shapiro, E. S., \& Kazak, A. E. (2003). Promoting Children's Health: Integrating School, Family, and Community. New York: Guilford Press.

Remuzzi, G., \& Garattini, S. (2008). Rare diseases: What's next? The Lancet, 371, 1978-1979.

Rezazadeh, S. M., \& Shaw, S. (2010). Rare chromosomal Disorders. In McCabe \& Shaw (Eds.), Genetic and Acquired Disorders: Current Topics and Interventions for Educators (pp. 20-28). Thousand Oaks, CA: Corwin.

Schieppati, A., Henter, J.-I., Daina, E., \& Aperia, A. (2008). Why rare diseases are an important medical and social issue. Lancet, 371, 2039-2041.

Shaw, S. R., Glaser, S. E., Stern, M., Sferdenschi, C., \& McCabe, P. C. (2010). Responding to Students' Chronic Illnesses. Principal Leadership, 10 (7), 12-16.

Shiu, S. (2001). Issues in the Education of Students with Chronic Illness. International Journal of Disability, Development and Education, 48 (3), 269-281.

Shiu, S. (2005). Enhancing the educational experience for children with chronic illness - What parents want educators to know. Acedido em 1 de dezembro de 2011, em : 
http://www.awch.org.au/pdfs/conferences/2005/06_SHIU,\%20Shiona _paper.pdf

Skinner, D., \& Schaffer, R. (2006). Families and Genetic Diagnoses in the Genomic and Internet Age. Infants \& Young Children, 19 (1), 16-24.

Tarniceru, A., Puiu, M., Cerbu, C. A., \& Serban, M. (2007). Rare diseases - a major problem of public health. Jurnalul Pediatrului, 10 (39-40), 3-6.

Taruscio, D., \& Cerbo, M. (1999). Malattie Rare: concetti generali, problem specifici et interventi sanitari. Ann. Est. Super. Sanità, 35 (2), 237-244.

Tavares, P. (1996). O aconselhamento genético. In L. Archer, J. Biscaia, \& W. Osswald, Bioética (pp. 261-265). Lisboa - São Paulo: Editorial Verbo.

Thies, K. M. (1999). Identifying the educational implications of chronic illness in school children. Journal of School Health, 69 (10), 392397.

Weely, S., \& Leufkens, H. G. (2004). Background Paper on Orphan Diseases for the WHO Report on Priority Medicines for Europe and the World. Acedido em 21 de fevereiro de 2012, em: http://www.pharmaceuticalpolicy.nl/Publications/Reports/7.5\%20Orp han\%20diseases.pdf

Wulffaert, J. (2010). Genetic Syndromes in the Family - Child characteristics and Parenting Stress in Angelman, CHARGE, Cornelia de Lange, Prader-Willi, and Rett syndrome. Doctoral Dissertation. Department of Clinical Child and Adolescent Studies, Faculty of Social and Behavioural Sciences, Leiden University.

Yaneva-Deliverska, M. (2011). Rare diseases and genetic discrimination. Journal of International Medical Association Bulgaria, 17 (1), pp. 116-119.

Zagalo-Cardoso, J. A. (2001). Doenças genéticas: impacto psicológico e representações sociais. In M. C. Canavarro (Org.), Psicologia da Gravidez e da Maternidade (pp. 211-235). Coimbra: Quarteto Editora.

Zurynski, Y., Frith, K., Leonard, H., \& Elliott, E. (2008). Rare childhood diseases: how should we respond? Archives of diseases in childhood, 93, 1071-1074.

\section{Legislação:}


Decreto-Lei n. ${ }^{\circ}$ 3/2008, de 7 de janeiro. (2008). Diário da República $n .^{\circ} 4$ - I Série. Lisboa: Ministério da Educação.

\section{Internet:}

www.eurordis.org, acedido em 2011-12-01

www.orpha.net, acedido em 2011-12-31

www.rarediseases.org, acedido em 2011-12-01

www.rarissimas.pt, acedido em 2011-12-01 